Copyright (C) 2013 IEEE. Personal use of this material is permitted. Permission from IEEE must be obtained for all other uses, in any current or future media, including reprinting/republishing this material for advertising or promotional purposes, creating new collective works, for resale or redistribution to servers or lists, or reuse of any copyrighted component of this work in other works. 


\title{
MMSE Based Transceiver Design for MIMO Relay Systems with Mean and Covariance Feedback
}

\author{
Lenin Gopal*, Yue Rong ${ }^{\dagger}$ and Zhuquan Zang* \\ *Department of Electrical and Computer Engineering, \\ Curtin University, Miri, 98009, Sarawak, Malaysia \\ Email: lenin@curtin.edu.my, zqzang@curtin.edu.my \\ ${ }^{\dagger}$ Department of Electrical and Computer Engineering, \\ Curtin University, Bentley, WA 6102, Australia \\ Email: y.rong@ @urtin.edu.au
}

\begin{abstract}
In this paper, the problem of transceiver design in a non-regenerative MIMO relay system is addressed, where linear signal processing is applied at the source, relay and destination to minimize the mean-squared error (MSE) of the signal waveform estimation at the destination. In the proposed design scheme, optimal structure of the source and relay precoding matrices are obtained with the assumption that the relay knows the mean and channel covariance information (CCI) of the relay-destination link and the full channel state information (CSI) of the sourcerelay link. Based on this assumption, an iterative joint source and relay precoder design is proposed to achieve the minimum MSE of the signal estimation at the destination. In order to reduce computational complexity of the proposed iterative design, a suboptimal relay-only precoder design is proposed. A numerical example shows that the performance of the proposed iterative joint source and relay precoder design is very close to that of the algorithm using full CSI.
\end{abstract}

\section{INTRODUCTION}

Recently cooperative communication has attracted considerable attention, due to its potential to provide reliable, cost effective and wide-area coverage of wireless networks. In cooperative communication systems, relay node can be deployed in between the source and destination to reduce the transmission power from the source to neighbouring nodes and mitigate the shadowing effects.

In general there are two kinds of relay strategies, including regenerative scheme and non-regenerative scheme [1]-[2]. In terms of implementation complexity, the non-regenerative scheme has a lower computational complexity, since for this scheme, the relay node amplifies the received signal from the source node and retransmits the signal to the destination node.

On the other hand, multiple antennas can provide spacial diversity and multiplexing gains to wireless communication systems. This benefits can be incorporated into the cooperative communication systems by deploying multiple antennas at the transceiver. Due to this fact, non-regenerative multiple input multiple output (MIMO) relay systems have received much research interest [1]-[11].

Recently, relay precoding scheme [1] and [2] for nonregenerative MIMO relaying has been investigated to maximize the capacity between the source and destination with further signal processing. In this scheme, the relay multiplies the received signal by a precoding matrix and retransmits

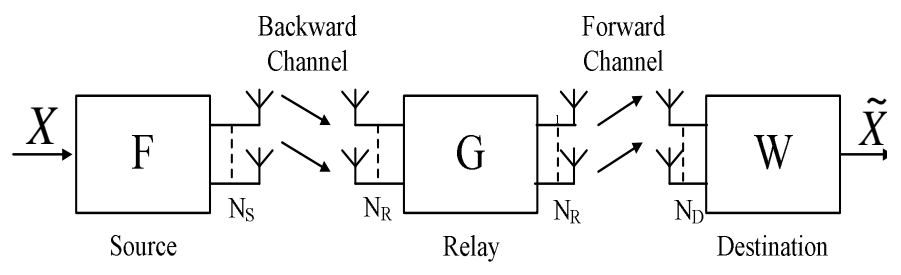

Fig. 1. Non-regenerative MIMO relay system

the precoded signal to the destination node. In [3]-[6], the precoding matrix was designed to minimize the MSE of the signal waveform estimation at the destination node. The optimal precoding matrix design was investigated well in [3]-[6] for non-regenerative MIMO relay system with the assumption that the relay knows the full channel state information (CSI) of the source-relay and relay-destination links.

In a practical system with a limited feedback rate, the assumption that the relay knows the full CSI for the relaydestination link is not feasible, especially in the situation when the destination node is moving rapidly. The channel mean and covariance matrices are more stable than the instantaneous channel matrix because the scattering environment changes more slowly compared to the destination node location.

In [7] and [8], optimal precoder is designed for maximizing the ergodic capacity of the non-regenerative MIMO relay systems with the assumption that the channel covariance information (CCI) of the relay-destination link is available at the relay node. Recently, minimum mean-squared error (MMSE) based transceivers are investigated in [9]-[11] with the assumption that the relay knows the covariance channel information of the relay-destination link. However, the optimal precoding matrix with the mean feedback of the relay-destination link was not investigated in [9]-[11].

In this paper, an iterative joint source and relay precoder design is proposed to minimize the MSE of the symbol estimation in a non-regenerative MIMO relay system, when the mean and covariance information for the relay-destination link is available at the relay. Considering that the computational complexity of the developed iterative scheme may be 
high for practical implementation of the relay system, we propose a suboptimal relay-only precoder design scheme. In the proposed two algorithms, it is assumed that the relay knows the full CSI of the source-relay link and mean and channel covariance information $(\mathrm{CCI})$ of the relay-destination link. Simulation results verify the performance of the proposed iterative and suboptimal mean and covariance based schemes.

\section{System Model And Problem Formulation}

Consider the non-regenerative MIMO relay system as shown in Fig.1, where the source, relay and destination have $N_{S}, N_{R}$ and $N_{D}$ antennas, respectively. It is assumed that there is no direct link between the source and destination due to long distance between these two nodes. The data transmission takes place over two hops. The received signal at the relay during the first hop is given by

$$
\mathbf{y}_{1}=\mathbf{H}_{1} \mathbf{F x}+\mathbf{n}_{1}
$$

where $\mathbf{H}_{1} \in \mathbb{C}^{N_{R} \times N_{S}}$ is the channel matrix of the sourcerelay link, $\mathbf{F} \in \mathbb{C}^{N_{S} \times N_{S}}$ is the source precoding matrix, $\mathbf{x} \in \mathbb{C}^{N_{S} \times 1}$ is the transmitted signal vector with covariance matrix $E\left\{\mathbf{x x}^{H}\right\}=\sigma_{x}^{2} \mathbf{I}_{N_{S}}, \mathbf{n}_{1} \in \mathbb{C}^{N_{R} \times 1}$ is the circularly symmetric complex Gaussian noise vector with zero mean and covariance matrix $E\left\{\mathbf{n}_{1} \mathbf{n}_{1}^{H}\right\}=\sigma_{1}^{2} \mathbf{I}_{N_{R}}$. Here $E\{$.$\} denotes the$ statistical expectation and (. $)^{H}$ stands for the matrix Hermitian transpose.

The received signal at the destination in the second hop is given by

$$
\mathbf{y}_{2}=\mathbf{H}_{2} \mathbf{G H}_{1} \mathbf{F x}+\mathbf{H}_{2} \mathbf{G n}_{1}+\mathbf{n}_{2}
$$

where $\mathbf{H}_{2} \in \mathbb{C}^{N_{D} \times N_{R}}$ is the channel matrix of the relaydestination link, $\mathbf{G} \in \mathbb{C}^{N_{R} \times N_{R}}$ is the relay precoding matrix, $\mathbf{n}_{2} \in \mathbb{C}^{N_{D} \times 1}$ is the circularly symmetric complex Gaussian noise vector with zero mean and covariance matrix $E\left\{\mathbf{n}_{2} \mathbf{n}_{2}^{H}\right\}=\sigma_{2}^{2} \mathbf{I}_{N_{D}}$. Let us introduce

$$
\mathbf{H}=\mathbf{H}_{2} \mathbf{G H}_{1} \mathbf{F}
$$

and

$$
\mathbf{n}=\mathbf{H}_{2} \mathbf{G} \mathbf{n}_{1}+\mathbf{n}_{2}
$$

where $\mathbf{H} \in \mathbb{C}^{N_{D} \times N_{S}}$ is the equivalent MIMO channel matrix, and $\mathbf{n} \in \mathbb{C}^{N_{D} \times 1}$ represents the equivalent noise vector. Now (2) can be written as

$$
\mathbf{y}_{2}=\mathbf{H} \mathbf{x}+\mathbf{n}
$$

Consider a scenario that the channel of the relay-destination link is correlated at the transmit antennas and is uncorrelated at the receive antennas. This model is suitable for an environment where the relay is not surrounded by local scatterers [12] and the destination node is hindered by local scatterers [8]. With this assumption, the channel matrix $\mathbf{H}_{2}$ can be modeled as

$$
\mathbf{H}_{2}=\overline{\mathbf{H}}_{\mu}+\mathbf{H}_{\omega} \boldsymbol{\Sigma}^{1 / 2}
$$

where $\overline{\mathbf{H}}_{\mu} \in \mathbb{C}^{N_{D} \times N_{R}}$ is the mean of $\mathbf{H}_{2}, \mathbf{H}_{\omega}$ is an $N_{D} \times N_{R}$ Gaussian matrix having i.i.d. circularly symmetric complex entries with zero mean and unit variance, and $\boldsymbol{\Sigma}$ is an $N_{R} \times N_{R}$ covariance matrix of $\mathbf{H}_{2}$ at the relay side.

At destination node, linear receiver $\mathbf{W}$ is applied to reduce implementation complexity. Hence, the estimated signal at the destination node can be expressed as

$$
\tilde{\mathbf{x}}=\mathbf{W H x}+\mathbf{W n} \text {. }
$$

We assume that the average power at the source and relay are upper bounded by $P_{s}$ and $P_{r}$. Since the transmitted signal from the relay is $\mathbf{G y}_{1}=\mathbf{G H}_{1} \mathbf{F} \mathbf{x}+\mathbf{G n}_{1}$, the power constraint on the source and relay can be expressed as

$$
\begin{gathered}
p(\mathbf{F})=\sigma_{x}^{2} \operatorname{tr}\left\{\mathbf{F}^{H} \mathbf{F}\right\} \leq P_{s} \\
p(\mathbf{F}, \mathbf{G})=\operatorname{tr}\left\{\mathbf{G}\left(\sigma_{x}^{2} \mathbf{H}_{1} \mathbf{F} \mathbf{F}^{H} \mathbf{H}_{1}^{H}+\sigma_{1}^{2} \mathbf{I}_{N_{R}}\right) \mathbf{G}^{H}\right\} \leq P_{r}
\end{gathered}
$$

where $\operatorname{tr}\{$.$\} is the trace of a matrix. Our goal is to design \mathbf{F}, \mathbf{G}$ and $\mathbf{W}$ so as to obtain the estimated signal which minimizes the following MSE function subject to the power constraints (8).

$$
J(\mathbf{F}, \mathbf{G}, \mathbf{W})=\operatorname{tr}\left\{E\left\{(\tilde{\mathbf{x}}-\mathbf{x})(\tilde{\mathbf{x}}-\mathbf{x})^{H}\right\}\right\}
$$

Mathematically, this problem can be formulated as

$$
\begin{aligned}
(\mathbf{F}, \mathbf{G}, \mathbf{W}) & =\underset{(\mathbf{F}, \mathbf{G}, \mathbf{W})}{\arg \min } J(\mathbf{F}, \mathbf{G}, \mathbf{W}), \\
\text { s.t. } p(\mathbf{F}) & \leq P_{s}, \\
p(\mathbf{F}, \mathbf{G}) & \leq P_{r} .
\end{aligned}
$$

After substituting (7) into (9), the MSE function (9) is simplified to

$$
\begin{aligned}
J(\mathbf{F}, \mathbf{G}, \mathbf{W})= & \operatorname{tr}\left\{\sigma_{x}^{2}\left(\mathbf{W H}-\mathbf{I}_{N_{S}}\right)\left(\mathbf{W H}-\mathbf{I}_{N_{S}}\right)^{H}\right. \\
& \left.+\mathbf{W R}_{n} \mathbf{W}^{H}\right\}
\end{aligned}
$$

where $\mathbf{R}_{n}$ is the equivalent noise covariance matrix, given by

$$
\mathbf{R}_{n}=\sigma_{1}^{2} \mathbf{H}_{2} \mathbf{G G}^{H} \mathbf{H}_{2}^{H}+\sigma_{2}^{2} \mathbf{I}_{N_{D}} .
$$

Note that directly solving the constrained MSE function (10) is difficult due to the fact that both the objective function $J(\mathbf{F}, \mathbf{G}, \mathbf{W})$ and the power constraint $p(\mathbf{F}, \mathbf{G})$ are non-linear and non-convex function of $\mathbf{F}, \mathbf{G}$ and $\mathbf{W}$.

In the following section a suboptimal approach will be used to tackle the constrained non-linear optimization problem. First, the problem will be solved for the optimal linear receiver $\mathbf{W}$ for any given precoding matrices $\mathbf{F}$ and $\mathbf{G}$ which satisfies the power constraints (8). Then, an iterative source and relay precoder design is proposed for obtaining the source and relay precoding matrices $\mathbf{F}$ and $\mathbf{G}$ by solving a closely related constrained optimization problem. In order to reduce computational complexity of the proposed iterative scheme, a suboptimal relay-only precoder design is proposed. 


\section{Optimal Transceiver Design}

For any given precoding matrices $\mathbf{F}$ and $\mathbf{G}$ which satisfy the power constraint at the source and relay nodes (8), the optimal linear receiver $\mathbf{W}$ that minimizes the MSE function $J(\mathbf{F}, \mathbf{G}, \mathbf{W})$ is the same as the MMSE (Wiener) receiver [13], which is given by

$$
\mathbf{W}=\sigma_{x}^{2} \mathbf{H}^{H}\left(\sigma_{x}^{2} \mathbf{H} \mathbf{H}^{H}+\mathbf{R}_{n}\right)^{-1} .
$$

After substituting (13) into (11), the MSE function is obtained as

$$
J(\mathbf{F}, \mathbf{G})=\sigma_{x}^{2} \operatorname{tr}\left\{\mathbf{I}_{N_{S}}-\sigma_{x}^{2} \mathbf{H}^{H}\left(\sigma_{x}^{2} \mathbf{H} \mathbf{H}^{H}+\mathbf{R}_{n}\right)^{-1} \mathbf{H}\right\} .
$$

Using the following matrix inversion lemma [13]

$$
\begin{aligned}
(\mathbf{A}+\mathbf{B C D})^{-1} & =\mathbf{A}^{-1}-\mathbf{A}^{-1} \mathbf{B} \\
& \times\left(\mathbf{D A} \mathbf{A}^{-1} \mathbf{B}+\mathbf{C}^{-1}\right)^{-1} \mathbf{D} \mathbf{A}^{-1},
\end{aligned}
$$

the MSE function (14) can be written as

$$
J(\mathbf{F}, \mathbf{G})=\sigma_{x}^{2} \operatorname{tr}\left\{\left[\mathbf{I}_{N_{S}}+\sigma_{x}^{2} \mathbf{H}^{H} \mathbf{R}_{n}^{-1} \mathbf{H}\right]^{-1}\right\} .
$$

Substituting (3) and (12) into (16), the MSE function can be expressed as

$$
\begin{aligned}
J(\mathbf{F}, \mathbf{G}) & =\sigma_{x}^{2} \operatorname{tr}\left\{\left[\mathbf{I}_{N_{S}}+\sigma_{x}^{2} \mathbf{F}^{H} \mathbf{H}_{1}^{H} \mathbf{G}^{H} \mathbf{H}_{2}^{H}\right.\right. \\
& \times\left(\sigma_{1}^{2} \mathbf{H}_{2} \mathbf{G G}^{H} \mathbf{H}_{2}^{H}+\sigma_{2}^{2} \mathbf{I}_{N_{D}}\right)^{-1} \\
& \left.\left.\times \mathbf{H}_{2} \mathbf{G H}_{1} \mathbf{F}\right]^{-1}\right\} .
\end{aligned}
$$

Using the matrix inversion lemma (15), the MSE function (17) can be written as

$$
\begin{aligned}
& J(\mathbf{F}, \mathbf{G})=\sigma_{x}^{2} \operatorname{tr}\left\{\left[\mathbf{I}_{N_{S}}+\frac{\sigma_{x}^{2}}{\sigma_{1}^{2}} \mathbf{F}^{H} \mathbf{H}_{1}^{H}\left[\mathbf{I}_{N_{R}}\right.\right.\right. \\
& \left.\left.\left.-\left(\mathbf{I}_{N_{R}}+\frac{\sigma_{1}^{2}}{\sigma_{2}^{2}} \mathbf{G}^{H} \mathbf{H}_{2}^{H} \mathbf{H}_{2} \mathbf{G}\right)^{-1}\right] \mathbf{H}_{1} \mathbf{F}\right]^{-1}\right\} .
\end{aligned}
$$

Now the problem is reduced to find the optimal precoder matrices $\mathbf{F}$ and $\mathbf{G}$ that minimize $J(\mathbf{F}, \mathbf{G})$ subject to the power constraints (8). Let us introduce the singular value decomposition (SVD) of $\mathbf{H}_{1}$

$$
\mathbf{H}_{1}=\mathbf{U}_{1} \boldsymbol{\Lambda}_{1}^{1 / 2} \mathbf{V}_{1}^{H}
$$

where $\boldsymbol{\Lambda}_{1}=\operatorname{diag}\left\{\Lambda_{1,1} \cdots \Lambda_{1, N_{R}}\right\}$ is a diagonal matrix with $\Lambda_{1,1} \geq \cdots \geq \Lambda_{1, N_{R}}, \mathbf{U}_{1}$ and $\mathbf{V}_{1}$ are the singular matrices of $\mathbf{H}_{1}$. To diagonalize (18), $\mathbf{F}$ can be selected as [14]

$$
\mathbf{F}=\mathbf{V}_{1} \boldsymbol{\Lambda}_{F}^{1 / 2} \mathbf{U}_{F}
$$

where $\boldsymbol{\Lambda}_{F}=\operatorname{diag}\left\{\Lambda_{\mathrm{F}, 1} \cdots \Lambda_{F, N_{R}}\right\}$ is a diagonal matrix with $\Lambda_{\mathrm{F}, 1} \geq \cdots \geq \Lambda_{F, N_{R}}$ and $\mathbf{U}_{F}$ is a unitary matrix. The eigenvalue decomposition of $\boldsymbol{\Sigma}$ can be expressed as

$$
\boldsymbol{\Sigma}=\mathbf{V}_{\Sigma} \boldsymbol{\Lambda}_{\Sigma} \mathbf{V}_{\Sigma}^{H}
$$

where $\boldsymbol{\Lambda}_{\Sigma}=\operatorname{diag}\left\{\Lambda_{\Sigma, 1} \cdots \Lambda_{\Sigma, N_{S}}\right\}$ with $\Lambda_{\Sigma, 1} \geq \cdots \geq$ $\Lambda_{\Sigma, N_{S}}$. The columns of $\mathbf{V}_{\Sigma}$ are the eigenvectors of $\boldsymbol{\Sigma}$ for the corresponding eigenvalues. Substituting (21) into (6), the channel matrix $\mathbf{H}_{2}$ can be written as

$$
\mathbf{H}_{2} \triangleq \overline{\mathbf{H}}_{\mu}+\widetilde{\mathbf{H}}_{\omega} \boldsymbol{\Lambda}_{\Sigma}^{1 / 2} \mathbf{V}_{\Sigma}^{H}
$$

where $\widetilde{\mathbf{H}}_{\omega} \triangleq \mathbf{H}_{\omega} \mathbf{V}_{\Sigma}$. Here, $\widetilde{\mathbf{H}}_{\omega}$ has the same distribution as $\mathbf{H}_{\omega}$, because the unitary matrix $\mathbf{V}_{\Sigma}$ does not change the statistical distribution of $\mathbf{H}_{\omega}$. Due to the similar statistical distribution, the $\widetilde{\mathbf{H}}_{\omega}$ is an $N_{D} \times N_{R}$ Gaussian matrix having i.i.d. circularly symmetric complex entries. Let's assume that the optimal precoding matrix $\mathbf{G}$ which minimizes (18) can be expressed as

$$
\mathbf{G}=\mathbf{V}_{\Sigma} \boldsymbol{\Lambda}_{G}^{1 / 2} \mathbf{U}_{1}^{H}
$$

where $\boldsymbol{\Lambda}_{G}=\operatorname{diag}\left\{\Lambda_{G, 1} \cdots \Lambda_{G, N_{R}}\right\}$. Substituting (19)-(23) in (18), the MSE function can be simplified to

$$
\begin{aligned}
J(\mathbf{F}, \mathbf{G}) & =\sigma_{x}^{2} \operatorname{tr}\left\{\left[\mathbf{I}_{N_{S}}+\frac{\sigma_{x}^{2}}{\sigma_{1}^{2}} \boldsymbol{\Lambda}_{F}^{1 / 2} \boldsymbol{\Lambda}_{1}^{1 / 2}\right.\right. \\
& \left.\left.\times\left[\mathbf{I}_{N_{R}}-\mathbf{D}_{1}\right] \boldsymbol{\Lambda}_{1}^{1 / 2} \boldsymbol{\Lambda}_{F}^{1 / 2}\right]^{-1}\right\}
\end{aligned}
$$

where

$$
\begin{aligned}
& \mathbf{D}_{1}=\left(\mathbf{I}_{N_{R}}+\frac{\sigma_{1}^{2}}{\sigma_{2}^{2}} \boldsymbol{\Lambda}_{G}^{1 / 2} \mathbf{V}_{\Sigma}^{H}\left[\overline{\mathbf{H}}_{\mu}^{H} \overline{\mathbf{H}}_{\mu}+\overline{\mathbf{H}}_{\mu}^{H} \widetilde{\mathbf{H}}_{\omega} \boldsymbol{\Lambda}_{\Sigma}^{1 / 2} \mathbf{V}_{\Sigma}^{H}\right.\right. \\
& \left.\left.+\mathbf{V}_{\Sigma} \boldsymbol{\Lambda}_{\Sigma}^{1 / 2} \widetilde{\mathbf{H}}_{\omega}^{H} \overline{\mathbf{H}}_{\mu}+\mathbf{V}_{\Sigma} \boldsymbol{\Lambda}_{\Sigma}^{1 / 2} \widetilde{\mathbf{H}}_{\omega}^{H} \widetilde{\mathbf{H}}_{\omega} \boldsymbol{\Lambda}_{\Sigma}^{1 / 2} \mathbf{V}_{\Sigma}^{H}\right] \mathbf{V}_{\Sigma} \boldsymbol{\Lambda}_{G}^{1 / 2}\right)^{-1} .
\end{aligned}
$$

It can be seen from (24) that $J(\mathbf{F}, \mathbf{G})$ depends on $\widetilde{\mathbf{H}}_{\omega}$, which is random and unknown. In the following, we optimize $E_{\widetilde{\mathbf{H}}_{\omega}}\{J(\mathbf{F}, \mathbf{G})\}$, where $E_{\widetilde{\mathbf{H}}_{\omega}}\{$.$\} indicates that the expecta-$ tion is taken with respect to the random matrix $\widetilde{\mathbf{H}}_{\omega}$. Now $E_{\widetilde{\mathbf{H}}_{\omega}}\{J(\mathbf{F}, \mathbf{G})\}$ can be expressed as

$$
\begin{aligned}
E_{\widetilde{\mathbf{H}}_{\omega}}\{J(\mathbf{F}, \mathbf{G})\} & =\sigma_{x}^{2} \sigma_{1}^{2} E_{\widetilde{\mathbf{H}}_{\omega}}\left[\operatorname { t r } \left\{\left[\sigma_{1}^{2} \mathbf{I}_{N_{S}}+\sigma_{x}^{2} \boldsymbol{\Lambda}_{F}^{1 / 2} \boldsymbol{\Lambda}_{1}^{1 / 2}\right.\right.\right. \\
& \left.\left.\left.\times\left[\mathbf{I}_{N_{R}}-\mathbf{D}_{1}\right] \boldsymbol{\Lambda}_{1}^{1 / 2} \boldsymbol{\Lambda}_{F}^{1 / 2}\right]^{-1}\right\}\right] .
\end{aligned}
$$

Now the work is left to determine the diagonal elements $\boldsymbol{\Lambda}_{F}$ and $\boldsymbol{\Lambda}_{G}$ of precoder matrices $\mathbf{F}$ and $\mathbf{G}$. Direct minimization of (25) for the optimal power allocation is difficult. In the following, the lower bound of the MSE is used together with the power constraint (8) to derive the suboptimal power allocation for the precoder matrices $\mathbf{F}$ and $\mathbf{G}$. Since $J(\mathbf{F}, \mathbf{G})$ is convex in $\widetilde{\mathbf{H}}_{\omega}^{H} \widetilde{\mathbf{H}}_{\omega}$, which is proved in [9], $E_{\widetilde{\mathbf{H}}_{\omega}}\{J(\mathbf{F}, \mathbf{G})\}$ has the following lower bound using Jensen's inequality [16]

$$
\begin{aligned}
J_{L}(\mathbf{F}, \mathbf{G}) & =\sigma_{x}^{2} \sigma_{1}^{2} \operatorname{tr}\left\{\left[\sigma_{1}^{2} \mathbf{I}_{N_{S}}+\sigma_{x}^{2} \boldsymbol{\Lambda}_{F}^{1 / 2} \boldsymbol{\Lambda}_{1}^{1 / 2}\right.\right. \\
& \left.\left.\times\left[\mathbf{I}_{N_{R}}-\mathbf{D}_{2}\right] \boldsymbol{\Lambda}_{1}^{1 / 2} \boldsymbol{\Lambda}_{F}^{1 / 2}\right]^{-1}\right\}
\end{aligned}
$$

where

$$
\begin{aligned}
\mathbf{D}_{2} & =\left(\mathbf{I}_{N_{R}}+\frac{\sigma_{1}^{2}}{\sigma_{2}^{2}} \boldsymbol{\Lambda}_{G}^{1 / 2} \mathbf{V}_{\Sigma}^{H}\left(\overline{\mathbf{H}}_{\mu}^{H} \overline{\mathbf{H}}_{\mu}\right.\right. \\
& +E_{\widetilde{\mathbf{H}}_{\omega}}\left\{\overline{\mathbf{H}}_{\mu}^{H} \widetilde{\mathbf{H}}_{\omega}\right\} \boldsymbol{\Lambda}_{\Sigma}^{1 / 2} \mathbf{V}_{\Sigma}^{H}+\mathbf{V}_{\Sigma} \boldsymbol{\Lambda}_{\Sigma}^{1 / 2} E_{\widetilde{\mathbf{H}}_{\omega}}\left\{\widetilde{\mathbf{H}}_{\omega}^{H} \overline{\mathbf{H}}_{\mu}\right\} \\
& \left.\left.+\mathbf{V}_{\Sigma} \boldsymbol{\Lambda}_{\Sigma}^{1 / 2} E_{\widetilde{\mathbf{H}}_{\omega}}\left\{\widetilde{\mathbf{H}}_{\omega}^{H} \widetilde{\mathbf{H}}_{\omega}\right\} \boldsymbol{\Lambda}_{\Sigma}^{1 / 2} \mathbf{V}_{\Sigma}^{H}\right) \mathbf{V}_{\Sigma} \boldsymbol{\Lambda}_{G}^{1 / 2}\right)^{-1}
\end{aligned}
$$

Using the properties of Gaussian random matrices with i.i.d circularly symmetric complex entries, $E_{\widetilde{\mathbf{H}}_{\omega}}\left\{\widetilde{\mathbf{H}}_{\omega}^{H} \widetilde{\mathbf{H}}_{\omega}\right\}=$ 
$N_{D} \mathbf{I}_{N R}, E_{\widetilde{\mathbf{H}}_{\omega}}\left\{\overline{\mathbf{H}}_{\mu}^{H} \widetilde{\mathbf{H}}_{\omega}\right\}=E_{\widetilde{\mathbf{H}}_{\omega}}\left\{\widetilde{\mathbf{H}}_{\omega}^{H} \overline{\mathbf{H}}_{\mu}\right\}=0$ and taking the expectation on (26) with respect to $E_{\widetilde{\mathbf{H}}_{\omega}}$, the MSE function can be simplified to

$$
\begin{aligned}
J_{L}(\mathbf{F}, \mathbf{G}) & =\sigma_{x}^{2} \sigma_{1}^{2} \operatorname{tr}\left\{\left[\sigma_{1}^{2} \mathbf{I}_{N_{S}}+\sigma_{x}^{2} \boldsymbol{\Lambda}_{F}^{1 / 2} \boldsymbol{\Lambda}_{1}^{1 / 2}\right.\right. \\
& \left.\left.\times\left[\mathbf{I}_{N_{R}}-\mathbf{D}_{3}\right] \boldsymbol{\Lambda}_{1}^{1 / 2} \boldsymbol{\Lambda}_{F}^{1 / 2}\right]^{-1}\right\}
\end{aligned}
$$

where

$$
\mathbf{D}_{3}=\left(\mathbf{I}_{N_{R}}+\frac{\sigma_{1}^{2}}{\sigma_{2}^{2}} \boldsymbol{\Lambda}_{G}^{1 / 2}\left(\mathbf{V}_{\Sigma}^{H} \overline{\mathbf{H}}_{\mu}^{H} \overline{\mathbf{H}}_{\mu} \mathbf{V}_{\Sigma}+N_{D} \boldsymbol{\Lambda}_{\Sigma}\right) \boldsymbol{\Lambda}_{G}^{1 / 2}\right)^{-1} .
$$

To proceed further, using the matrix inversion lemma (15), the MSE function (27) can be written as

$$
\begin{aligned}
J_{L}(\mathbf{F}, \mathbf{G}) & =\sigma_{x}^{2} \operatorname{tr}\left\{\left[\mathbf{I}_{N_{S}}-\frac{1}{\sigma_{1}^{2}} \boldsymbol{\Lambda}_{F}^{1 / 2} \boldsymbol{\Lambda}_{1}^{1 / 2} \boldsymbol{\Lambda}_{G}^{1 / 2}\right.\right. \\
& \times\left(\frac{1}{\sigma_{1}^{2}} \boldsymbol{\Lambda}_{G}^{1 / 2} \boldsymbol{\Lambda}_{1}^{1 / 2} \boldsymbol{\Lambda}_{F} \boldsymbol{\Lambda}_{1}^{1 / 2} \boldsymbol{\Lambda}_{G}^{1 / 2}+\mathbf{C}\right)^{-1} \\
& \left.\left.\times \boldsymbol{\Lambda}_{G}^{1 / 2} \boldsymbol{\Lambda}_{1}^{1 / 2} \boldsymbol{\Lambda}_{F}^{1 / 2}\right]\right\}
\end{aligned}
$$

where

$$
\mathbf{C}=\frac{1}{\sigma_{x}^{2}}\left[\boldsymbol{\Lambda}_{G}+\frac{\sigma_{2}^{2}}{\sigma_{1}^{2}}\left(\mathbf{V}_{\Sigma}^{H} \overline{\mathbf{H}}_{\mu}^{H} \overline{\mathbf{H}}_{\mu} \mathbf{V}_{\Sigma}+N_{D} \boldsymbol{\Lambda}_{\Sigma}\right)^{-1}\right] .
$$

We consider an upper-bound of (28) as follows. Then the MSE function (28) can be rewritten as

$$
\begin{aligned}
J_{L}(\mathbf{F}, \mathbf{G}) & =\sigma_{x}^{2}\left[\operatorname{tr}\left(\mathbf{A}^{-1}\right)-\operatorname{tr}\left(\mathbf{A}^{-1} \mathbf{E}^{H}\right.\right. \\
& \left.\left.\times\left(\mathbf{E} \mathbf{A}^{-1} \mathbf{E}^{H}+\mathbf{C}\right)^{-1} \mathbf{E A}^{-1}\right)\right]
\end{aligned}
$$

where

$$
\begin{aligned}
& \mathbf{A}=\mathbf{I}_{N_{S}}, \\
& \mathbf{E}=\frac{1}{\sigma_{1}} \boldsymbol{\Lambda}_{G}^{1 / 2} \boldsymbol{\Lambda}_{1}^{1 / 2} \boldsymbol{\Lambda}_{F}^{1 / 2} .
\end{aligned}
$$

By using the following inequality from [14]

$$
\begin{aligned}
& \operatorname{tr}\left(\mathbf{A}^{-1} \mathbf{E}^{H}\left(\mathbf{E} \mathbf{A}^{-1} \mathbf{E}^{H}+\mathbf{C}\right)^{-1} \mathbf{E} \mathbf{A}^{-1}\right) \\
& \geq \operatorname{tr}\left(\mathbf{A}^{-1} \mathbf{E}^{H}\left(\mathbf{E} \mathbf{A}^{-1} \mathbf{E}^{H}+\operatorname{diag}(\mathbf{C})\right)^{-1} \mathbf{E} \mathbf{A}^{-1}\right)
\end{aligned}
$$

where $\operatorname{diag}(\mathbf{C})$ is obtained from $\mathbf{C}$ by setting its off-diagonal elements to zero and an upper-bound of $J_{L}(\mathbf{F}, \mathbf{G})$ is given by

$$
\begin{aligned}
J_{U}(\mathbf{F}, \mathbf{G}) & =\sigma_{x}^{2} \operatorname{tr}\left\{\left[\mathbf{I}_{N_{S}}-\left(\boldsymbol{\Lambda}_{F} \boldsymbol{\Lambda}_{1} \boldsymbol{\Lambda}_{G}+\sigma_{1}^{2} \boldsymbol{\Lambda}_{C}\right)^{-1}\right.\right. \\
& \left.\left.\times \boldsymbol{\Lambda}_{F} \boldsymbol{\Lambda}_{1} \boldsymbol{\Lambda}_{G}\right]\right\}
\end{aligned}
$$

where

$$
\begin{aligned}
\boldsymbol{\Lambda}_{C} & =\frac{1}{\sigma_{x}^{2}} \operatorname{diag}\left[\boldsymbol{\Lambda}_{G}+\frac{\sigma_{2}^{2}}{\sigma_{1}^{2}}\right. \\
& \left.\times\left(\mathbf{V}_{\Sigma}^{H} \overline{\mathbf{H}}_{\mu}^{H} \overline{\mathbf{H}}_{\mu} \mathbf{V}_{\Sigma}+N_{D} \boldsymbol{\Lambda}_{\Sigma}\right)^{-1}\right] .
\end{aligned}
$$

Inserting (20) into (8), the power constraint for the source node can be expressed as

$$
p(\mathbf{F})=\sigma_{x}^{2} \operatorname{tr}\left\{\boldsymbol{\Lambda}_{F}\right\} \leq P_{s} .
$$

Substituting (19) and (23) into (8), the power constraint for the relay node can be expressed as

$$
p(\mathbf{F}, \mathbf{G})=\operatorname{tr}\left\{\left(\sigma_{x}^{2} \boldsymbol{\Lambda}_{1} \boldsymbol{\Lambda}_{F}+\sigma_{1}^{2} \mathbf{I}_{N_{R}}\right) \boldsymbol{\Lambda}_{G}\right\} \leq P_{r} .
$$

\section{A. Joint Source and Relay Precoder Design}

In this section, a joint source and relay procder design is proposed to obtain the diagonal elements of $\boldsymbol{\Lambda}_{F}, \boldsymbol{\Lambda}_{G}$. From (31), (33) and (34), the diagonal elements of $\boldsymbol{\Lambda}_{F}, \boldsymbol{\Lambda}_{G}$ can be obtained by solving the following constrained optimization problem with scalar variables

$$
\begin{gathered}
\min J_{U}(\mathbf{F}, \mathbf{G})=\sum_{i=1}^{N_{S}} \frac{\sigma_{x}^{2} \sigma_{1}^{2} \Lambda_{C, i}}{\Lambda_{1, i} \Lambda_{F, i} \Lambda_{G, i}+\sigma_{1}^{2} \Lambda_{C, i}} \\
\text { s.t. } p(\mathbf{F})=\sigma_{x}^{2} \sum_{i=1}^{N_{S}} \Lambda_{F, i} \leq P_{s}, \\
p(\mathbf{F}, \mathbf{G})=\sum_{i=1}^{N_{S}}\left(\sigma_{x}^{2} \Lambda_{1, i} \Lambda_{F, i}+\sigma_{1}^{2}\right) \Lambda_{G, i} \leq P_{r} .
\end{gathered}
$$

Using the Karush-Kuhn-Tucker (KKT) conditions [15], the optimal diagonal elements of $\Lambda_{F, i}$ and $\Lambda_{G, i}$ are obtained as

$$
\begin{aligned}
& \Lambda_{F, i}=\frac{1}{\Lambda_{1, i} \Lambda_{G, i}}\left(\sqrt{\frac{\sigma_{1}^{2} \Lambda_{C, i} \Lambda_{1, i} \Lambda_{G, i}}{\mu_{s}+\mu_{r} \Lambda_{1, i} \Lambda_{G, i}}}-\sigma_{1}^{2} \Lambda_{C, i}\right)^{+} \\
& \Lambda_{G, i}=\frac{1}{\Lambda_{1, i} \Lambda_{F, i}}\left(\sqrt{\frac{\sigma_{x}^{2} \sigma_{1}^{2} \Lambda_{C, i} \Lambda_{1, i} \Lambda_{F, i}}{\mu_{r}\left(\sigma_{x}^{2} \Lambda_{1, i} \Lambda_{F, i}+\sigma_{1}^{2}\right)}}-\sigma_{1}^{2} \Lambda_{C, i}\right)^{+}
\end{aligned}
$$

where $(x)^{+}=\max (x, 0), \mu_{s}$ and $\mu_{r}$ should be chosen to meet the power constraints (36) and (37).

It can be seen from (38) and (39) that $\Lambda_{F, i}, \Lambda_{G, i}$ are function of each other, so directly solving the diagonal elements of the matrices are difficult. To avoid this difficulty, an iterative algorithm is proposed to compute the diagonal elements of $\boldsymbol{\Lambda}_{F}$ and $\boldsymbol{\Lambda}_{G}$.

In this algorithm, initialize $\boldsymbol{\Lambda}_{F}=\mathbf{I}_{N_{s}}$ and $\boldsymbol{\Lambda}_{G}=\mathbf{I}_{N_{s}}$. Then calculate $\boldsymbol{\Lambda}_{C}$ with (32), and calculate the water filling variables $\mu_{r}$ and $\mu_{s}$ to satisfy the power constraints (8) at the source and destination nodes. Update $\boldsymbol{\Lambda}_{F}$ and $\boldsymbol{\Lambda}_{G}$ according to (38) and (39) respectively. $\boldsymbol{\Lambda}_{F}$ and $\boldsymbol{\Lambda}_{G}$ are iteratively updated until $\left\|\boldsymbol{\Lambda}_{F}^{\prime}-\boldsymbol{\Lambda}_{F}\right\|^{2} \leq 0.0001$ and $\left\|\boldsymbol{\Lambda}_{G}^{\prime}-\boldsymbol{\Lambda}_{G}\right\|^{2} \leq 0.0001$. Here, $\boldsymbol{\Lambda}_{F}^{\prime}$ and $\boldsymbol{\Lambda}_{G}^{\prime}$ are the two recent calculated values of $\boldsymbol{\Lambda}_{F}$ and $\boldsymbol{\Lambda}_{G}$ and $\|\cdot\|^{2}$ denotes the squared Frobenius norm.

\section{B. Relay-only Precoder Design}

In this section, we propose a suboptimal algorithm to obtain the diagonal elements of $\boldsymbol{\Lambda}_{G}$ while fixing $\boldsymbol{\Lambda}_{F}$. Let us assume that $\boldsymbol{\Lambda}_{F}=\mathbf{I}_{N_{S}}$, the the constrained optimization problem (35)-(37) can be rewritten in scalar form as

$$
\begin{gathered}
\min J_{U}(\mathbf{G})=\sum_{i=1}^{N_{S}} \frac{\sigma_{x}^{2} \sigma_{1}^{2} \Lambda_{C, i}}{\Lambda_{1, i} \Lambda_{G, i}+\sigma_{1}^{2} \Lambda_{C, i}} \\
\text { s.t. } p(\mathbf{F})=\sigma_{x}^{2} N_{S} \leq P_{s}, \\
p(\mathbf{G})=\sum_{i=1}^{N_{S}}\left(\sigma_{x}^{2} \Lambda_{1, i}+\sigma_{1}^{2}\right) \Lambda_{G, i} \leq P_{r} .
\end{gathered}
$$




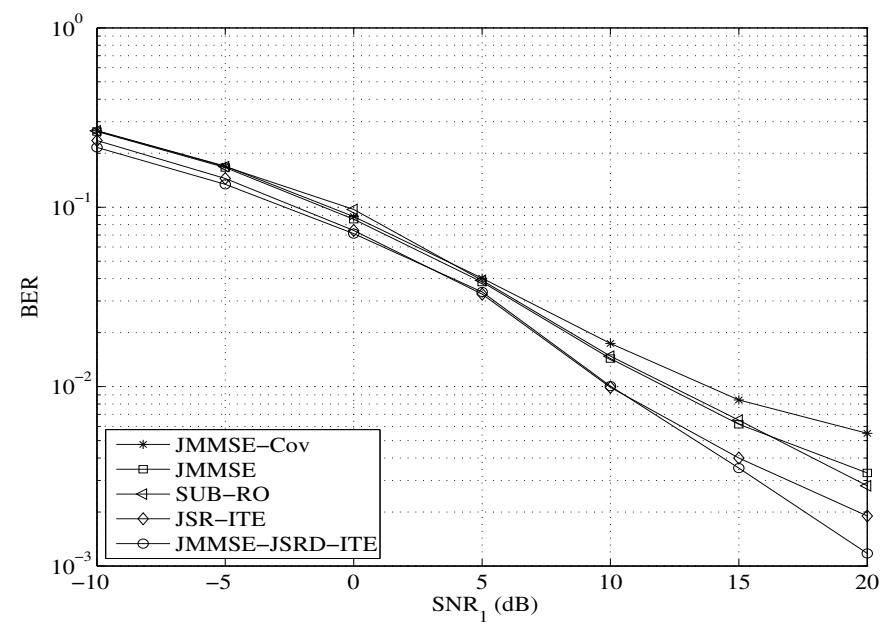

Fig. 2. BER versus $S N R_{1}$ while fixing $S N R_{2}=20 d B$.

Using the KKT conditions [15], the optimal diagonal elements of $\Lambda_{G, i}$ are obtained as

$$
\Lambda_{G, i}=\frac{1}{\Lambda_{1, i}}\left(\sqrt{\frac{\sigma_{x}^{2} \sigma_{1}^{2} \Lambda_{C, i} \Lambda_{1, i}}{\mu_{r}\left(\sigma_{1}^{2} \Lambda_{1, i}+\sigma_{1}^{2}\right)}}-\sigma_{1}^{2} \Lambda_{C, i}\right)^{+}
$$

where $\mu_{r}$ should be chosen to meet the power constraint (42).

\section{Simulation Results}

In this section, we study the performance of the proposed schemes by a numerical example. We simulate the nonregenerative MIMO relay system with $N_{S}=N_{R}=N_{D}=4$. The unitary matrix $\mathbf{U}_{F}$ of the source precoder matrix (20) is generated by the $N_{S}$-point discrete Fourier-transform matrix. The channel matrices $\mathbf{H}_{1}$ and $\mathbf{H}_{\omega}$ are generated as complex Gaussian variables with zero mean and unit variance and the symbols are generated from QPSK constellation.

The mean, $\overline{\mathbf{H}}_{\mu}$, of $\mathbf{H}_{2}$ is randomly generated. The elements of covariance matrix $\boldsymbol{\Sigma}$ of $\mathbf{H}_{2}$ is generated by $\Sigma_{i, j}=$ $j_{0}(\triangle \pi|i-j|)$ [12], where $j_{0}($.$) is the zeroth order Bessel$ function of the first kind, $\triangle$ the angle of fading spread. We consider the angle spread as $\triangle=30^{\circ}$. The SNRs for the source-relay and relay-destination links are defined as follows $S N R_{1}=\frac{\sigma_{x}^{2}}{\sigma_{1}^{2}}, S N R_{2}=\frac{P_{r}}{N_{R} \sigma_{1}^{2}}$.

We compare the performance of the proposed schemes with the joint MMSE scheme (JMMSE) [3], joint MMSE covariance scheme (JMMSE-Cov) [10], and the iterative joint source, relay and destination scheme (JMMSE-JSRD-ITE) [6]. The JMMSE-JSRD-ITE scheme provides the lower-bound of the proposed schemes.

Fig.2 shows the performance of the proposed MMSE schemes in terms of BER versus $S N R_{1}$ while fixing $S N R_{2}=$ $20 \mathrm{~dB}$. The proposed two relay schemes, suboptimal relay-only (SUB-RO) scheme and iterative joint source and relay (JSRITE) scheme, show better BER performance over all range of $S N R_{1}$ than the JMMSE-Cov scheme. For all range of $S N R_{1}$, the BER performance of the SUB-RO scheme is closer to that of the JMMSE scheme. The proposed JSR-ITE scheme outperforms the JMMSE-Cov, SUB-RO and JMMSE schemes over the tested range of $S N R_{1}$.

\section{CONCLUSiON}

In this paper, we have proposed an iterative joint source and relay precoder design scheme to minimize the MSE of the symbol estimation at the destination with the assumption that the mean and covariance feedback of the relay-destination link is available at the relay. We assumed that the relay knows the full CSI of the source-relay link. Due to the computational complexity of the proposed iterative scheme, a suboptimal relay-only precoder scheme is proposed. Simulation results show that the proposed iterative and suboptimal schemes minimize the upper-bound of the MSE and it is demonstrated that the proposed schemes have better performance in terms of BER as compared to the conventional covariance feedback based MSE schemes.

\section{REFERENCES}

[1] O. Muñoz-Medina, J. Vidal, and A. Agustín, "Linear transceiver design in non-regenerative relays with channel state information," IEEE Trans. Signal Process., vol. 55, pp. 2593 - 2604, June 2007.

[2] X. Tang and Y. Hua, "Optimal design of non-regenerative MIMO wireless relays," IEEE Trans. Wireless Commun., vol. 6, pp. 1398 - 1407, Apr. 2007.

[3] W. Guan and H. Luo, "Joint MMSE transceiver design in non-regenerative MIMO relay systems," IEEE Commun. Lett., vol. 12, pp. 517 - 519, July 2008.

[4] A. S. Behbahani, R. Merched, and A. M. Eltawil, "Optimizations of a MIMO relay network," IEEE Trans. Signal Process., vol. 56, pp. 5062 5073, Oct. 2008.

[5] Y. Rong, X. Tang, and Y. Hua, "A unified framework for optimizing linear non-regenerative multicarrier MIMO relay communication systems," IEEE Trans. Signal Process., vol. 57, pp. 4837-4851, Dec. 2009.

[6] B. Zhang, X. Wang, K. Niu, and Z. He, "Joint linear transceiver design for non-regenerative MIMO relay systems," Electronics Letters, vol. 45, pp. 1254-1256, Nov. 2009.

[7] H. W. Je, B. Lee, S. Kim, and K. B. Lee, "Design of non-regenerative MIMO-relay systems with partial channel state information," in Proc IEEE Int. Conf. Commun, Beijing, 2008, pp. 4441-4445.

[8] C. Jeong and H.-M. Kim, "Precoder design of non-regenerative relays with covariance feedback," IEEE Commun. Lett., vol. 13, no.12, pp.920 - 922, Dec. 2009.

[9] K. Dae-Hyun and H. -M. Kim, "MMSE precoder design for a nonregenerative MIMO relay with covariance feedback," in Proc. IEEE 21st Int. Sym. Per. Ind. Mobile Radio Commun., Istanbul, Turkey, pp. 461-464, Aug. 2010.

[10] L. Gopal, Y. Rong, and Z. Zang, "Joint MMSE transceiver design in nonregenerative MIMO relay systems with covariance feedback", in Proc. IEEE 17th Asia-Pacific Conf. Commun., Sabah, Malaysia, Oct. 2011.

[11] L. Gopal, Y. Rong, and Z. Zang, "Channel covariance information based transceiver design for AF MIMO relay systems with direct link", in Proc. IEEE 18th Asia-Pacific Conf. Commun., Korea, Oct. 2012.

[12] D. Shiu, G. J. Foschini, M. J. Gans, and J. M. Kahn, "Fading correlation and its effect on the capacity of multielement antenna systems," IEEE Trans. Commun., vol. 48, no. 3, pp. 502-513, Mar. 2000.

[13] S. M. Kay, "Fundamentals of Statistical Signal Processing: Estimation Theory," Englewood Cilffs, NJ: Prentice Hall, 1993.

[14] F. S. Tseng and W. R. Wu, "Linear MMSE transceiver design in amplifyand-and-forward MIMO relay systems," IEEE Trans. Veh. Technol., vol. 59, no. 2, pp. 754-765, Feb. 2010.

[15] E. K. P. Chong and S. H. Zak, "Introduction to optimization," New York, NY:Wiley-Interscience, 2001.

[16] A. W. Marshall, I. Olkin and B. C. Arnold, "Inequalities: Theory of majorization and its applications," New York, Springer, 2011. 